\title{
Response and representation of ductile damage under varying shock loading conditions in tantalum
}

DOI:

$10.1063 / 1.4941823$

\section{Document Version}

Final published version

Link to publication record in Manchester Research Explorer

\section{Citation for published version (APA):}

Bronkhorst, C. A., Gray, G. T., Addessio, F. L., Livescu, V., Bourne, N. K., MacDonald, S. A., \& Withers, P. J. (2016). Response and representation of ductile damage under varying shock loading conditions in tantalum. Journal of Applied Physics, 119(8), [085103]. https://doi.org/10.1063/1.4941823

\section{Published in:}

Journal of Applied Physics

\section{Citing this paper}

Please note that where the full-text provided on Manchester Research Explorer is the Author Accepted Manuscript or Proof version this may differ from the final Published version. If citing, it is advised that you check and use the publisher's definitive version.

\section{General rights}

Copyright and moral rights for the publications made accessible in the Research Explorer are retained by the authors and/or other copyright owners and it is a condition of accessing publications that users recognise and abide by the legal requirements associated with these rights.

\section{Takedown policy}

If you believe that this document breaches copyright please refer to the University of Manchester's Takedown Procedures [http://man.ac.uk/04Y6Bo] or contact uml.scholarlycommunications@manchester.ac.uk providing relevant details, so we can investigate your claim.

\section{OPEN ACCESS}




\section{AIP $\left.\right|_{\substack{\text { Applied Physics } \\ \text { Joumal of }}}$}

\section{Response and representation of ductile damage under varying shock loading conditions in tantalum}

C. A. Bronkhorst, G. T. Gray III, F. L. Addessio, V. Livescu, N. K. Bourne, S. A. McDonald, and P. J. Withers

Citation: Journal of Applied Physics 119, 085103 (2016); doi: 10.1063/1.4941823

View online: http://dx.doi.org/10.1063/1.4941823

View Table of Contents: http://scitation.aip.org/content/aip/journal/jap/119/8?ver=pdfcov

Published by the AIP Publishing

\section{Articles you may be interested in}

Nucleation and growth of damage in polycrystalline aluminum under dynamic tensile loading AIP Advances 5, 037116 (2015); 10.1063/1.4914919

Analysis of deformation twinning in tantalum single crystals under shock loading conditions

J. Appl. Phys. 113, 083522 (2013); 10.1063/1.4792227

Roughness of Ductile Damage Paths in Shock Loaded Tantalum

AIP Conf. Proc. 845, 670 (2006); 10.1063/1.2263411

Delayed failure in a shock-loaded silicon carbide

J. Appl. Phys. 97, 113513 (2005); 10.1063/1.1923161

The Deviatoric Response of Three Dense Glasses under Shock Loading Conditions AIP Conf. Proc. 620, 807 (2002); 10.1063/1.1483660

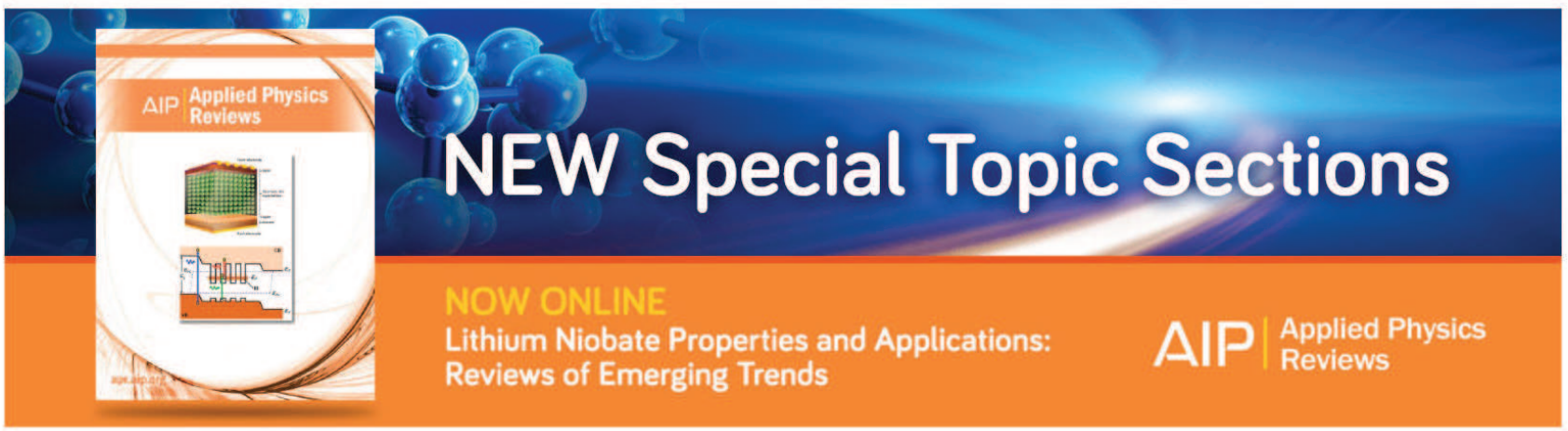




\title{
Response and representation of ductile damage under varying shock loading conditions in tantalum
}

\author{
C. A. Bronkhorst, ${ }^{1, \text { a) }}$ G. T. Gray III, ${ }^{1}$ F. L. Addessio, ${ }^{1}$ V. Livescu, ${ }^{1}$ N. K. Bourne ${ }^{2}$ \\ S. A. McDonald, ${ }^{2}$ and P. J. Withers ${ }^{2}$ \\ ${ }^{1}$ Los Alamos National Laboratory, Los Alamos, New Mexico 87545, USA \\ ${ }^{2}$ School of Materials, University of Manchester, Manchester M13 9PL, United Kingdom
}

(Received 9 October 2015; accepted 30 January 2016; published online 25 February 2016; corrected 2 March 2016)

\begin{abstract}
The response of polycrystalline metals, which possess adequate mechanisms for plastic deformation under extreme loading conditions, is often accompanied by the formation of pores within the structure of the material. This large deformation process is broadly identified as progressive with nucleation, growth, coalescence, and failure the physical path taken over very short periods of time. These are well known to be complex processes strongly influenced by microstructure, loading path, and the loading profile, which remains a significant challenge to represent and predict numerically. In the current study, the influence of loading path on the damage evolution in high-purity tantalum is presented. Tantalum samples were shock loaded to three different peak shock stresses using both symmetric impact, and two different composite flyer plate configurations such that upon unloading the three samples displayed nearly identical "pull-back" signals as measured via rear-surface velocimetry. While the "pull-back" signals observed were found to be similar in magnitude, the sample loaded to the highest peak stress nucleated a connected field of ductile fracture which resulted in complete separation, while the two lower peak stresses resulted in incipient damage. The damage evolution in the "soft" recovered tantalum samples was quantified using optical metallography, electron-back-scatter diffraction, and tomography. These experiments are examined numerically through the use of a model for shock-induced porosity evolution during damage. The model is shown to describe the response of the tantalum reasonably well under strongly loaded conditions but less well in the nucleation dominated regime. Numerical results are also presented as a function of computational mesh density and discussed in the context of improved representation of the influence of material structure upon macro-scale models of ductile damage. (C) 2016 AIP Publishing LLC.

[http://dx.doi.org/10.1063/1.4941823]
\end{abstract}

\section{INTRODUCTION}

Research over the past six decades has provided a wealth of experimental data and insight concerning the influence of microstructure and shock pulse history (i.e., peak stress, pulse duration, loading and release rates, and onedimensional versus complex loading) on the dynamic failure (spallation) response of metals. ${ }^{1-4}$ Investigations by Rinehart ${ }^{5}$ and Butcher et al. ${ }^{6}$ detailed how the formation and thickness of the "scabbing under explosive loading" (i.e., spallation), depends on the shape of the stress wave imposed. Butcher $e t a l .{ }^{6}$ demonstrated that "since spallation is studied by introducing a pulse and observing the effects of the tensile stresses within the material, knowledge of the complete stress history at the spall plane is necessary for the quantitative understanding of spall." Experiments by Butcher et al. ${ }^{6}$ and more recently by Gray et al. ${ }^{4}$ showed "that the spallation stress is less for square pulses than for triangular pulses" in metals displaying ductile damage evolution modes. Contrary to ductile metals where the kinetics for void initiation, growth, and linkage result in a strong dependence of spall strength on wave profile shape, the dynamic damage of tungsten heavy alloy (WHA), which fail by brittle cleavage

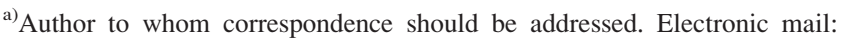
cabronk@lanl.gov
}

through the tungsten particles, is seen to be relatively insensitive to the shock pulse duration and accompanying unloading (tensile) pulse. ${ }^{7}$ In this case, the brittle damage kinetics and the spall strength were dominated by the shock peak stress, independent of the shock pulse duration. The influence of non-one-dimensional oblique loading paths has also been shown to significantly affect ductile damage evolution during spallation loading in copper. ${ }^{8}$ High-explosive-driven sweeping-wave loading of tantalum has been observed to yield lower spall strength than previously documented for one-dimensional supported-shock-wave loading and to exhibit increased shock hardening as a function of increasing obliquity. ${ }^{8}$

In tandem with the wealth of knowledge gained from experimental probing of materials under shock conditions, similar progress has been made in the ability to represent these rapid physical processes numerically. In particular, the high density tantalum material that is examined in this paper has been of interest for some time. ${ }^{9}$ The constitutive model used for this work began with the work of Johnson ${ }^{2}$ and the development of a constitutive model for shock loaded copper representing nonlinear elasticity, plasticity, and evolution of local porosity. Johnson ${ }^{2}$ recognized the significance of the aggregate nature of polycrystalline metallic materials and used a unit cell analysis of the elastic-plastic response of the 
material under rapidly loaded conditions. Inertial effects were recognized as important but were not included in the analysis of copper presented. The model was applied to problems of plate impact, explosively loaded material, and an expanding ring. Much later, this model was also applied to the study of loading pulse duration in copper. ${ }^{10}$ Addessio and Johnson ${ }^{11}$ improved upon the work of Johnson ${ }^{2}$ by proposing a modified Gurson ${ }^{12}$ type model, which also employed an overstress equation for the plastic flow rule of the material. This equation introduces a length scale into the series of equations and assists in regularizing the problem. This helps to alleviate issues of numerical stability and severe mesh sensitivity. The authors also clearly recognized the possibility that relying upon deformation rate sensitivity within the model would not always solve the regularization issue, but that additional length scales (e.g., spatial gradients in physically based internal state variables) may be required in general. A void nucleation model was not proposed and the model was tested against copper plate impact experiments. It was demonstrated that for the problems examined, the use of the overstress model reduced the mesh sensitivity of the simulated results.

The model of Addessio and Johnson ${ }^{11}$ was expanded upon by Maudlin et al. ${ }^{13}$ to include the effect of non-isotropic plastic flow. They also included the ability to account for the anisotropic nature of the void as it grows but this capability is not exercised in the work presented here. Significant advancements were made to the solution algorithm to improve the numerical efficiency of the solution. This model was used on several dynamic loading boundary value problems with success. The numerical algorithm portion of this work was later published by Zuo and Rice. ${ }^{14}$ They presented results of the influence of the overstress parameter on the shape of the flow stress surface under differing stress conditions.

The purpose of the current study was to subject tantalum samples to three different peak shock stresses followed by, as close as possible, nearly identical tensile loading pulses leading to spallation damage evolution. The results, as will be shown, demonstrate a strong difference in the loading profile for the three loading conditions and a significantly different damage field in the three identical material samples. The model of Addessio and Johnson, ${ }^{11}$ Maudlin et al., ${ }^{13}$ and Zuo and Rice ${ }^{14}$ was used to represent these experimental results. This paper begins by reporting upon the details of the experimental conditions used on the tantalum material. The experimental results are then presented and linked to the three different loading profiles used. This includes detailed examination of the cross-sectional state of the samples after loading. The constitutive model is then presented in overview form and the material parameters used are presented. The numerical models used to represent the three experimental conditions and simulation results are discussed. The paper concludes with a detailed discussion of the results and some final thoughts.

\section{EXPERIMENTAL TECHNIQUES}

\section{A. Material}

Targets for the spallation experiments were prepared from commercially pure, triple electron-beam melted, and annealed tantalum plate, $10.2 \mathrm{~mm}$ in thickness obtained from Cabot Corporation. ${ }^{15}$ The chemical composition (in wt. \%) was analyzed to be carbon $10 \mathrm{ppm}$, oxygen $<50 \mathrm{ppm}$, nitrogen $<10$ ppm, hydrogen $<5$ ppm, tungsten $<25$ ppm, niobium $<25 \mathrm{ppm}$, titanium $<5 \mathrm{ppm}$, iron $<5 \mathrm{ppm}$, and the balance tantalum. The plate was produced from an ingot, which was forged into a billet; this was then annealed, and subsequently cut prior to cross rolling. The plates were straight rolled in the final finishing passes. The texture strength was moderate, with the $\langle 111\rangle$ fiber component approximately three times random. Average grain size of this material was $35 \mu \mathrm{m}$. Acoustic properties of the tantalum were measured using $5 \mathrm{MHz}$ quartz transducers with a Parametrics 500PR pulse receiver. The density $\left(\rho_{0}\right)$ of the tantalum studied was measured to be $16.58 \pm 0.01 \mathrm{~g} / \mathrm{cc}$, longitudinal sound speed $\left(C_{L}\right) 4130 \pm 30 \mathrm{~m} / \mathrm{s}$, shear sound speed $\left(C_{S}\right) 2040 \pm 30 \mathrm{~m} / \mathrm{s}$, and Poisson ratio $(\nu)$ was 0.339 .

\section{B. Plate impact testing}

The primary diagnostics for these experiments was sample recovery with post-mortem metallurgical analysis and Photon Doppler Velocimetry (PDV). ${ }^{16}$ Soft recovered spall plate impact experiments were conducted using an $80 \mathrm{~mm}$ bore gas launcher and soft-recovery techniques presented previously. ${ }^{17}$ Three identical $4.572 \mathrm{~mm}$ thick right circular tantalum targets were prepared with press fit momentum trapping rings to mitigate perturbations from edge release waves. A square wave profile was achieved using a $2.25 \mathrm{~mm}$ thick tantalum monolithic impactor impacting the spall sample at $250 \mathrm{~m} / \mathrm{s}$. The thickness of this impactor was chosen as one-half that of the target to locate the spall plane at the target midline. Two higher peak stress spallation tests were conducted using composite impactors. The first composite impactor consisted of $1.27 \mathrm{~mm}$ of tantalum backed by $4.98 \mathrm{~mm}$ of aluminum and was launched into the target at $375 \mathrm{~m} / \mathrm{s}$, while the highest stress impactor consisted of $1.18 \mathrm{~mm}$ of copper backed by $1.24 \mathrm{~mm}$ of tungsten and launched into the target at $615 \mathrm{~m} / \mathrm{s}$. The composite flyers were fabricated by gluing together the different metals chosen, using a low-viscosity epoxy and clamped under pressure during curing, to achieve a thin uniform bond layer. The glue bonded layer was approximately $150 \mu \mathrm{m}$ thick. Impact velocities were measured to an accuracy of $1 \%$ using a sequential pressure transducer method and sample tilt was fixed to $\sim 1 \mathrm{mrad}$ by means of an adjustable specimen mount. Following impact, all samples were soft recovered by decelerating them into low-density foam. The free surface velocity (FSV) history profiles were measured using PDV singlepoint probes, one aimed at the sample center and one off center, and analyzed using the procedures of Jensen et al. ${ }^{16}$ Both PDV traces were found to be identical on each of the three tantalum spallation experiments.

Following loading, the tantalum specimens were crosssectioned and prepared for optical metallography and electron backscatter diffraction (EBSD). ${ }^{17,18}$ Automated EBSD scans were performed with a step size of $0.11 \mathrm{~mm}$ in a hexagonal grid at $20 \mathrm{kV}$ in an FEI XL30 FEG-SEM equipped with TSL data acquisition hardware and software. Analysis 
of EBSD data provided crystal orientation information, as well as spatial misorientation distribution associated with the void damage field. X-ray microtomography measurements were carried out using an Xradia MicroXCT which was equipped with a $150 \mathrm{keV}$ sealed tungsten high-energy microfocus X-ray source and a $2048 \times 2048$ pixel 16-bit high resolution cooled $\mathrm{CCD}$ detector. Owing to the high X-ray absorption of tantalum, small matchstick samples of crosssection $0.6 \times 0.6 \mathrm{~mm}^{2}$ were required to ensure adequate transmission of $\mathrm{X}$-rays in the projections. These were cut from the $10.6 \mathrm{GPa}$ shocked tantalum sample such that the long axis represented the thickness of the impacted target. The tomography figures were calculated by segmenting the reconstructed X-ray tomography volumes based on grey level, therefore separating the voids from the sample bulk. By selecting a single voxel within a void, all connected voxels to this, within a specified grey level range, are also automatically selected, ensuring the shape of the void to be defined by the boundary between the void and the metal phase. Applying this for each of the voids within the scanned region-of-interest (ROI), having a height of $1.1 \mathrm{~mm}$, results in a segmented volume of voids, this can be displayed as a three-dimensional surface plot.

\section{EXPERIMENTAL RESULTS}

\section{A. Free surface velocity profiles}

The free surface velocity (FSV) profiles for the experiments are shown in Fig. 1(a). These measurements were performed at the sample back free surface. All three loading profiles exhibit a pronounced Hugoniot Elastic Limit (HEL) at $\sim 50 \mathrm{~m} / \mathrm{s}$. The three spallation experiments reach peak shock stresses in the Ta targets of 7.4, 10.6, and $21 \mathrm{GPa}$, respectively, while displaying differing pulse durations of $\sim 0.84, \sim 0.28$, and $\sim 0.25 \mu \mathrm{s}$, respectively, as a consequence of the differing impactor designs utilized. The highest peak stress loaded spall sample $(21 \mathrm{GPa})$ resulted in full spallation, while the 7.4 and 10.6 peak stress tests resulted in incipient spall damage. The magnitude of the apparent "pullback" signals, denoted by the red arrowed lines superimposed on the FSV profiles, is however seen to be essentially identical for each of the three experiments, while the peak shock stresses and pulse durations at peak stress are substantially different. Utilizing the standard straight impedance spall drop relationship proposed by Novikov et al., ${ }^{19}$ where $\sigma_{\text {spall }}=\frac{1}{2} \rho_{0} c_{B}(\Delta F S V)$ yields spall strengths of 4.71, 4.70, and $5.20 \mathrm{GPa}$, respectively, for the three tantalum spall shots ( $\rho_{0}$ is the unloaded material density and $C_{B}$ is the bulk sound speed). For the highest peak stress test, $21 \mathrm{GPa}$, where complete sample separation occurred, the dynamic impedance can be corrected using $\sigma_{\text {spall }}=\frac{1}{2} \rho_{0} c_{B}(\Delta F S V+\delta)$ as proposed by $\mathrm{Kanel}^{20}$ resulting in a corrected spall strength of 4.85 ( $\delta$ is a wave interaction correction term due to the formation of damage regions).

\section{B. Post-mortem metallographic analysis}

The damage evolution in the three tantalum samples was seen to vary as a function of the varying peak shock

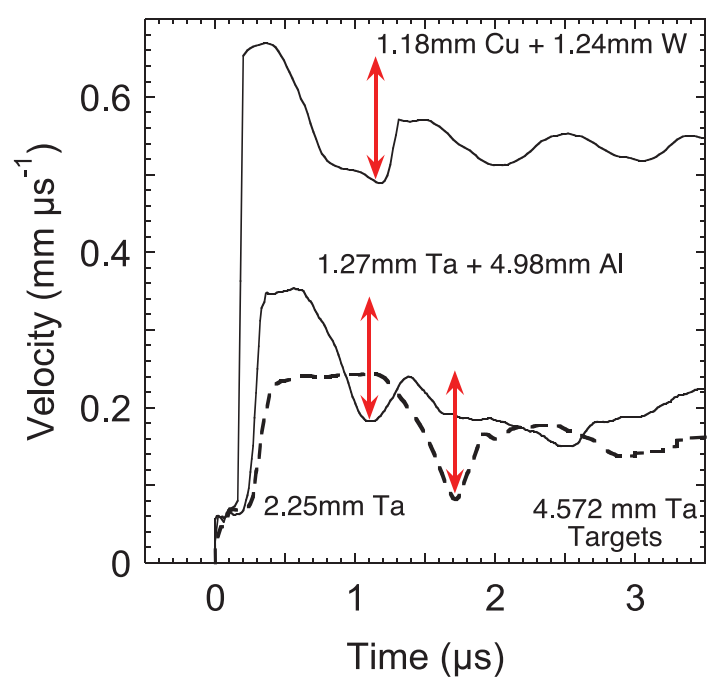

(a)

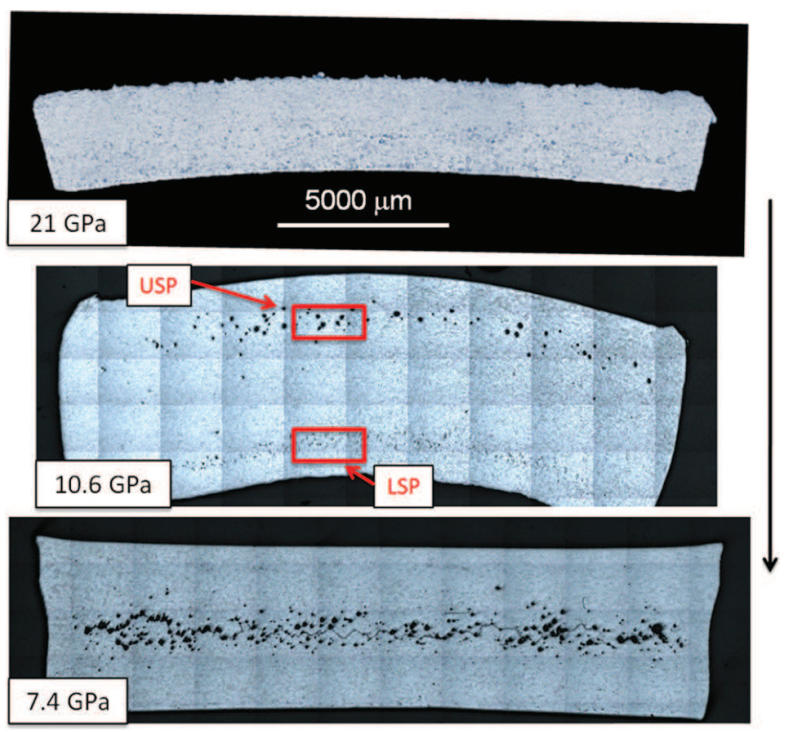

(b)

FIG. 1. (a) Traces of the free surface velocity for the three different peak stress/impactor configurations, and (b) macroscopic thru-thickness crosssectional views of the three Ta samples shocked to 7.4, 10.6, and $21 \mathrm{GPa}$. (Shock-loading direction as indicated by arrow in figure.)

stresses and pulse durations, while nominally exhibiting roughly similar "pull-back" signals. Figure 1(b) shows thruthickness macroscopic optical micrographs of the three tantalum spallation samples. The sample loaded to a peak shock stress of $21 \mathrm{GPa}$ is seen to have fully separated into two pieces (only the lower half is shown in Fig. 1(b)), while the 10.6 and 7.4 GPa loaded samples exhibit macroscopic incipient damage with a clear finite-width field. The tantalum on tantalum symmetrically impacted sample loaded to $7.4 \mathrm{GPa}$ displays a well-developed field of voids located mid-plane in the sample. A representative area showing very intense and complex localization linkages connecting the voids is shown in Fig. 2(a). Large lattice rotations of up to $45^{\circ}$ are evident in the grains adjacent to the linkages. Isolated fine twins are seen throughout the microstructure. The sample loaded with the composite impactor to $10.6 \mathrm{GPa}$ peak stress displays two incipient damage fields, termed the upper (USP) and lower 


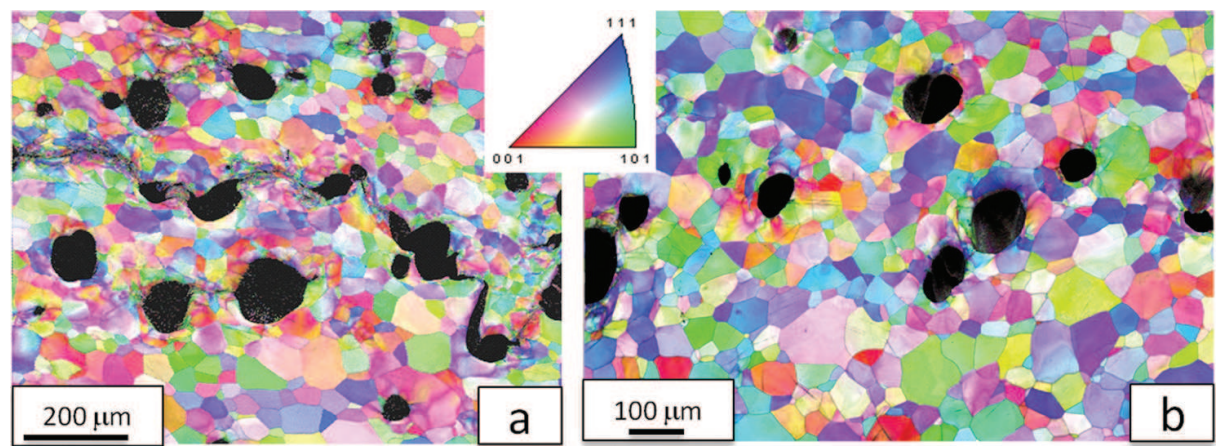

FIG. 2. Crystal direction map of (a) the $7.4 \mathrm{GPa}$ sample showing wellformed spherical voids with intense localization linkages between voids and (b) the $10.6 \mathrm{GPa}$ sample at the upper spall plane USP (see Figure 1(b)) showing voids with very few localization linkages from primary damage area.

(LSP) spall plane regions (Figs. 2(b), 3(a), and 3(b)). A field of isolated $\sim 50$ to $100 \mu \mathrm{m}$ sized voids located roughly $1 \mathrm{~mm}$ from the upper sample surface is seen in the upper spall plane of the sample and an additional field of numerous small $(<50 \mu \mathrm{m})$ void nucleation sites as seen in Fig. 3(a) is seen within $1 \mathrm{~mm}$ of the lower sample surface. The primary, upper spall plane, damage field displays very few localization linkages connecting the voids, isolated fine twins, and intragranular lattice rotations of up to $35^{\circ}$ in the grains adjacent to the voids. The lower damage field displays more extensive deformation throughout the region with most grains affected by deformation, no evidence of deformation twinning, extensive deformation near grain boundaries, and intragranular lattice rotations across the region of $35^{\circ}$ as seen in Figs. 3(a) and 3(b). Figure 3(a) provides a unique view into the details of void nucleation in the lower spall plane. The anisotropic distribution of dislocation slip bands/localized shear features all located at or near grain boundaries identifies grain boundaries as the preferred nucleation damage sites for incipient spall in these nominally onedimensional spall experiments on high-purity tantalum. Tomography of the upper and lower spall plane regions was found to exhibit voids consistent with the optical metallography, as seen in Figs. 4(a) and 4(b). Within the regions of interest selected, the void volume fractions were measured to be $1.23 \%$ and $0.08 \%$ within the upper and lower spall planes, respectively. The sample loaded to a peak shock stress of $21 \mathrm{GPa}$ displayed full spall with ductile fracture separating the sample into two pieces with plasticity and intense damage directly adjacent to the fracture surface as seen in Fig. 4(c). Some isolated small voids are seen at grain boundaries away from the fracture surface in addition to isolated deformation twins adjacent to the fracture surface. The zone of damage is concentrated within $500 \mu \mathrm{m}$ of the fracture surface and many of the voids were seen at grain boundaries and coalescence of these is effectively "unzipping" the material along the boundaries.

Taken collectively, the velocimetry and post-mortem metallurgical analysis of the spallation samples provides some interesting insights into the damage evolution and spallation mechanisms operative in tantalum for the shock drive imposed. To first order, the fact that the three different peak shock stresses when subjected to nearly identical tensile pulses resulted in essentially the same "pull-back" signals supports minimal peak-stress or rate-dependent strain hardening or shock hardening on the damage evolution in tantalum. This finding is consistent with the lack of ratedependent strain hardening as well as the lack of enhanced shock hardening previously linked to planar dislocation glide and the high Peierls-Nabarro stress in tantalum. ${ }^{21}$ Examination of the details of the damage evolution of the three spall samples illustrates that in tantalum under the imposed nominally one-dimensional loading: (1) initial damage nucleation occurs via anisotropic slip/shear at selected grain boundaries in a heterogeneous manner (Figs. 3(a) and 3(b)), (2) initial void growth leads to $\sim$ spherical voids (measured max to min average diameters in upper spall plane showed ratio of 1.66) reflecting the local hydrostatic loading, and (3) void coalescence in adjacent voids occurs via pronounced shear localization.

The observation of damage nucleation leading to voids located at grain boundaries in the current study is different than that seen in the case of void formation occurring preferentially at deformation twin-twin intersections or at twingrain boundary intersections ${ }^{8}$ in tantalum shocked via a sweeping detonation wave. The increased propensity for twin formation during sweeping-detonation-wave loading, at a peak shock stress nominally similar to the $10.6 \mathrm{GPa}$ current nominally one-dimensional tantalum sample loading, reinforces the importance of shock obliquity, and its
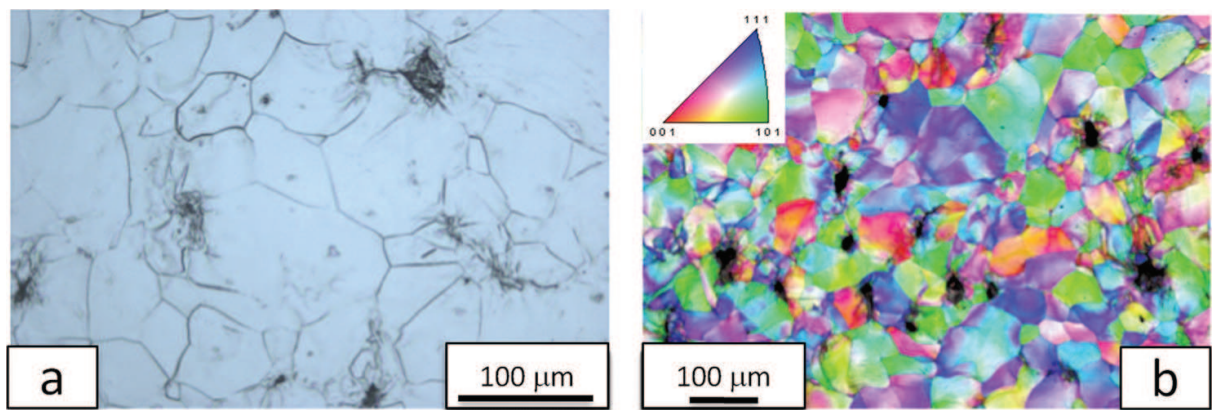

FIG. 3. Lower spall plane (LSP) $10.6 \mathrm{GPa}$ sample showing localized plasticity vortices within extensive deformation-affected grains serving as nucleation sites for small incipient voids as viewed using (a) optical metallography - etched sample, and (b) EBSD imaging. 

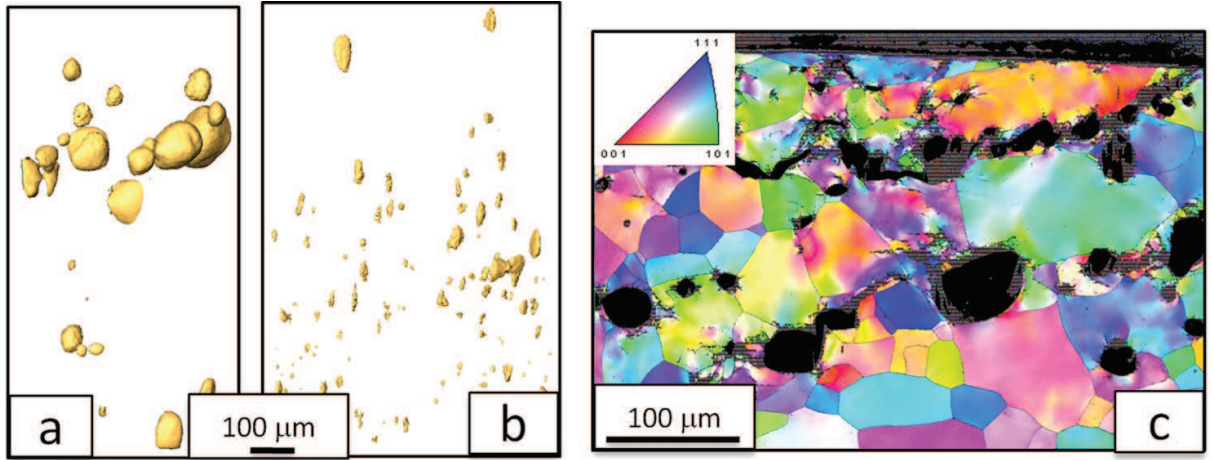

FIG. 4. (a) Tomography of voids in 10.6 GPa sample upper spall plane, and (b) tomography of lower spall plane in 10.6 GPa sample, (c) crystal direction map for the $21 \mathrm{GPa}$ sample exhibiting voids and extensive deformationaffected grains just below the complete fracture surface. commensurate higher shear stresses. It has been observed that with increased shock obliquity there is a greater propensity for deformation twin formation and thereafter damage nucleation mechanisms during spallation. Finally, the essentially identical "pull-back" signals, resulting from the three different shock-loading histories, which display different evolved damage fields, illustrates the lack of uniqueness of a given "pull-back" spall signal magnitude obtained from rearsurface velocimetry to quantifying shock-induced spall- and shock-induced damage evolution in materials.

\section{CONSTITUTIVE MODELING}

\section{A. Nomenclature}

Standard direct notation is used throughout this paper. Second rank tensors are denoted by bold face upper case letters. Fourth rank tensors are denoted by underscored bold face upper case letters. The following variables are used: I identity, F deformation gradient, D stretch, T Cauchy stress, $\rho$ density, and $\theta$ temperature. The prime symbol $\mathbf{A}^{\prime}$ indicates a deviatoric quantity. The inner product of two second rank tensors $\mathbf{A}$ and $\mathbf{B}$ is defined by $\mathbf{A} \cdot \mathbf{B}=\operatorname{trace}\left(\mathbf{A}^{\mathrm{T}} \mathbf{B}\right)$. The overtilde $\tilde{\mathbf{A}}$ represents the quantity $\mathbf{A}$ in the undamaged material.

\section{B. Constitutive model}

The constitutive model used in this study is derived from the work of Addessio and Johnson, ${ }^{11}$ Maudlin et al., ${ }^{13,22}$ and Zou. ${ }^{23}$ The model is applied to tantalum plate impact loading as presented earlier and is summarized here.

The Cauchy stress in the damaged state is given by

$$
\mathbf{T}=\underline{\mathbf{M}} \tilde{\mathbf{T}}
$$

where the stress in the undamaged material is $\tilde{\mathbf{T}}$ and the general fourth rank isotropic damage tensor is given by

$$
\underline{\mathbf{M}}=(1-\phi) \underline{\mathbf{I}},
$$

with the scalar internal state variable $\phi$ representing the isotropic state of porosity at the material point used in this study. In general, the tensor $\underline{\mathbf{M}}$ allows for the anisotropic representation of damage evolution within the material. However for the present study this dependence is assumed to remain isotropic. Time integration is performed in the unrotated frame relative to the current configuration defined by the rotation $\mathbf{R}$ given by the polar decomposition

$$
\mathbf{F}=\mathbf{R} \mathbf{U}=\mathbf{V R}
$$

The material time rate for the Cauchy stress defined in the unrotated frame relative to the current configuration is given by

$$
\begin{aligned}
\dot{\mathbf{T}}= & \underline{\mathbf{M}} \underline{\tilde{\mathbf{L}^{\prime}}}\left(\mathbf{D}^{\prime}-\mathbf{D}^{p^{\prime}}\right)+\underline{\mathbf{M}}\left(J \tilde{\mathbf{K}}_{s} \operatorname{tr} \mathbf{D}^{e}-\frac{\tilde{\rho}}{\rho} \tilde{\Gamma} \mathbf{T} \cdot \mathbf{D}^{p}\right) \underline{\mathbf{I}} \\
& +\underline{\dot{\mathbf{M}}} \underline{\mathbf{M}}^{-1} \mathbf{T},
\end{aligned}
$$

where $\underline{\tilde{\mathbf{L}}}$ is the fourth order elastic stiffness tensor, $J=\tilde{\rho}_{0} / \tilde{\rho}, \tilde{K}_{s}$ is the isentropic solid bulk modulus, $\tilde{\Gamma}$ is the Gruneisen coefficient, and

$$
\tilde{P}=-\frac{1}{3} \operatorname{tr} \tilde{\mathbf{T}}
$$

The deformation rate $\mathbf{D}$ is additively decomposed as

$$
\mathbf{D}=\mathbf{D}^{e}+\mathbf{D}^{p}=\mathbf{D}^{e}+\left(\mathbf{D}^{d}+\mathbf{D}^{p^{\prime}}\right)
$$

where the plastic contribution $\left(\mathbf{D}^{p}\right)$ to the rate of deformation is separated into spherical $\left(\mathbf{D}^{d}\right)$ and deviatoric $\left(\mathbf{D}^{p^{\prime}}\right)$ components. The spherical component and the contribution due to damage $^{11}$ is given by

$$
\dot{\phi}=(1-\phi) \operatorname{trD}^{d} .
$$

The plastic flow rule is given by

$$
\mathbf{D}^{p}=\frac{1}{\tau_{r}}\left(\mathbf{T}-\mathbf{T}^{p r o j}\right) .
$$

This over-stress style approach uses a relaxation constant $\tau_{r}$ with the tensorial quantity $\mathbf{T}^{\text {proj }}$ being the current Cauchy stress projected onto the plastic flow surface given below in Eq. (10). Based upon Addessio and Johnson ${ }^{11}$ and Maudlin et al., ${ }^{13}$ the approximate length scale implied by $\tau_{r}$ is given by

$$
l=\frac{\tau_{r}}{\sqrt{\rho_{0}\left(K+\frac{4}{3} G\right)}},
$$

where $\mathrm{K}$ and $\mathrm{G}$ are the ambient condition solid bulk and shear moduli, respectively.

Equation (4) contains four terms defining the time evolution of Cauchy stress, each with a specific physical significance. The first term is the contribution to the evolution of the 
stress due to deviatoric elastic deformation. The second term is due to influence of mean volumetric elastic deformation in the material given that finite elastic strains and substantial pressures must be accounted for. The third term is due to the thermal expansion or contraction of the material through the Gruneisen parameter and thermal energy produced via plastic work. The fourth and final term is due to the evolution of stress due to the effects of damage evolution.

Therefore, Eq. (8) allows for the possibility of states of stress external to the flow surface. The role of this additional viscous relationship and the selection of $\tau_{r}$ magnitude will be discussed in more depth below. The porosity modulated plastic flow surface employed here is that developed by Gurson $^{12}$ and extended by Addessio and Johnson ${ }^{11}$ and Maudlin, ${ }^{13}$ given by

$$
\tau-\sigma_{f}\left(\dot{\bar{\varepsilon}}_{p}, \theta\right)^{2}\left[1+q_{3} \phi^{2}-2 q_{1} \phi \cosh \delta\right]=0,
$$

where

$$
\tau=\frac{1}{2} \mathbf{T}^{\prime} \cdot \underline{\alpha} \mathbf{T}^{\prime}
$$

is a quadratic relationship allowing for plastic anisotropy using the anisotropy tensor $\underline{\boldsymbol{\alpha}}, \sigma_{f}\left(\dot{\bar{\varepsilon}}_{p}, \theta\right)$ is the rate and temperature sensitive scalar flow stress with

$$
\begin{gathered}
\dot{\bar{\varepsilon}}_{p}=\sqrt{\frac{2}{3} \mathbf{D}^{p^{\prime}} \cdot \mathbf{D}^{p^{\prime}}}, \\
\delta=-\frac{3 q_{2} \tilde{P}}{2 \sigma_{s}} .
\end{gathered}
$$

The quantities $q_{1}, q_{2}$, and $q_{3}$ are material parameters and the saturation flow stress $\sigma_{s}$ is defined below (Eq. (26)).

The criterion for computational cell failure as a function of porosity and plastic strain is a modified HancockMackenzie $^{24}$ relationship and is defined as

$$
F=\left(\frac{\phi}{\phi_{f}}\right)^{2}+\left(\frac{\bar{\varepsilon}_{p}}{\gamma_{f}}\right)^{2} \geq 1
$$

where $\bar{\varepsilon}_{p}=\int \dot{\bar{\varepsilon}}_{p} d t, \phi_{f}$ is the failure porosity, and

$$
\gamma_{f}=\gamma_{0}+\gamma_{1} e^{\gamma_{2} \frac{\tilde{P}}{\tau}}
$$

where $\tilde{P}$ is the tensile hydrostatic pressure and $\gamma_{0}, \gamma_{1}$, and $\gamma_{2}$ are material parameters evaluated from notched bar tensile experiments.

Equation (14) represents combined effects of porosity and plastic deformation so that when $F$ reaches a value of 1.0 , the material at that particular material point no longer retains load bearding ability. We only consider monotonic states of damage; re-compaction of damaged regions is not considered here. A polynomial Mie-Gruneisen equation of state is used for the volumetric component of compressive states of stress as a function of density

$$
\tilde{P}=\left(K_{1} \tilde{\beta}+K_{2} \tilde{\beta}^{2}+K_{3} \tilde{\beta}^{3}\right)(1-\tilde{\Gamma} \tilde{\beta} / 2)+\tilde{\Gamma} \tilde{E}_{s}(1+\tilde{\beta}),
$$

where

$$
\begin{gathered}
\tilde{\beta}=\frac{\tilde{\rho}}{\tilde{\rho}_{0}}-1 ; \\
\rho=(1-\phi) \tilde{\rho} . \\
\tilde{E}_{s}=\int\left(\bar{\sigma} \dot{\bar{\varepsilon}}-\tilde{P} \operatorname{tr}\left(\mathbf{D}^{e}+\mathbf{D}^{d}\right)\right) J d t \\
\bar{\sigma}=\sqrt{\frac{3}{2} \mathbf{T}^{\prime} \cdot \mathbf{T}^{\prime}}
\end{gathered}
$$

$K_{1}, K_{2}$, and $K_{3}$ are coefficients.

The rate and temperature sensitivity of the plastic deformation response is represented through the flow stress. The deformation of tantalum at rates observed here has been shown to be well represented by several constitutive models ${ }^{9,25-28}$ which are based upon the thermal activation kinetics developed by Kocks et al. ${ }^{29}$ We employ here the isotropic mechanical threshold stress (MTS) model, which has been well established for tantalum and is due to the work of Follansbee and Kocks, ${ }^{30}$ Chen and Gray, ${ }^{9}$ and Maudlin et al. ${ }^{22}$ The MTS model is based on the concept of a superposition of resistances to the glide of dislocations. Generally, they are grouped as athermal barriers (e.g., grain boundaries) and thermally influenced barriers (e.g., Peierls stress-intrinsic lattice resistance, forest dislocations, dislocation structure, and solute atoms). The mechanical threshold stress is the deformation resistance at $0 \mathrm{~K}$. The flow stress used here is the stress adjusted to current temperature and strain rate. The reader is referred to Follansbee and Kocks ${ }^{30}$ and Chen and Gray ${ }^{9}$ for more details.

The relationship for the solid material flow stress is given by

$$
\sigma_{f}\left(\dot{\bar{\varepsilon}}_{p}, \theta\right)=\sigma_{a}+\frac{\mu}{\mu_{0}}\left(S_{i}\left(\dot{\bar{\varepsilon}}_{p}, \theta\right) \hat{\sigma}_{i}+S_{\varepsilon}\left(\dot{\bar{\varepsilon}}_{p}, \theta\right) \hat{\sigma}_{\varepsilon}\right)
$$

where $\sigma_{a}$ is the constant athermal resistance, $\hat{\sigma}_{i}$ is the constant intrinsic lattice resistance at $0 \mathrm{~K}$, and $\hat{\sigma}_{\varepsilon}$ is the resistance due to dislocation structure at $0 \mathrm{~K}$, which evolves with deformation. The relationship for shear modulus as a function of temperature is given ${ }^{31}$ as

$$
\mu=\mu_{0}-\frac{D_{0}}{\exp \left(\frac{\theta_{0}}{\theta}\right)-1}
$$

The rate and temperature kinetics are represented by the two pre-multiplying terms

$$
S_{i}\left(\dot{\bar{\varepsilon}}_{p}, \theta\right)=\left(1-\left[\frac{k \theta}{\mu b^{3} g_{0 i}} \ln \left(\frac{\dot{\varepsilon}_{0 i}}{\dot{\bar{\varepsilon}}_{p}}\right)\right]^{\frac{1}{q_{i}}}\right)^{\frac{1}{p_{i}}}
$$

and

$$
S_{\varepsilon}\left(\dot{\bar{\varepsilon}}_{p}, \theta\right)=\left(1-\left[\frac{k \theta}{\mu b^{3} g_{0 \varepsilon}} \ln \left(\frac{\dot{\varepsilon}_{0 \varepsilon}}{\dot{\bar{\varepsilon}}_{p}}\right)\right]^{\frac{1}{q_{\varepsilon}}}\right)^{\frac{1}{p_{\varepsilon}}},
$$

and $k$ is Boltzmann's constant, $b$ is the magnitude of the Burgers vector, $g_{0}$ are normalized activation energies, $\dot{\varepsilon}_{0}$ are 
reference strain rates, and $p$ and $q$ are exponents, which determine the shape of the energy barrier profile. Kocks et $a .^{29}$ suggest that $p \in[0,1]$ and $q \in[1,2]$.

The resistance due to the evolution of the dislocation structure changes with strain as

$$
\frac{d \hat{\sigma}_{\varepsilon}}{d \bar{\varepsilon}_{p}}=h_{0}\left(1-\frac{\hat{\sigma}_{\varepsilon}}{\hat{\sigma}_{\varepsilon S}}\right)^{\kappa},
$$

where the saturation stress as a function of rate and temperature is given by ${ }^{32}$

$$
\hat{\sigma}_{\varepsilon S}=\hat{\sigma}_{\varepsilon S_{0}}\left(\frac{\dot{\bar{\varepsilon}}_{p}}{\dot{\varepsilon}_{0_{\varepsilon S}}}\right)^{\frac{k \theta}{\mu h^{3} g_{0 s s}}} .
$$

The saturation stress $\sigma_{s}$, used in Eq. (13), is taken as the current value of the flow stress given in Eq. (21) with the quantity $\hat{\sigma}_{\varepsilon}$ replaced by its saturation value $\hat{\sigma}_{\varepsilon s}$ given by Eq. (26).

The local mechanical work done to the material changes the local temperature by the following relationship:

$$
\dot{\theta}=\frac{1}{\rho C_{p}} \dot{\tilde{E}}_{s}
$$

where $\tilde{E}_{s}$ is the internal energy in the undamaged material (Eq. (19)) and $C_{p}$ is the specific heat at constant pressure.

As described above in the experimental section, tungsten, aluminum, and copper were used as flyer plates to achieve differing loading profiles. Each of these materials was represented through equation of state and plastic flow stress models. All three materials were represented with the Mie-Gruneisen form of equation of state as given above in Eqs. (16)-(20). The parameters for tungsten are $\rho_{0}$ $=16981.5 \mathrm{~kg} / \mathrm{m}^{3}, K_{1}=302.059 \mathrm{GPa}, K_{2}=469.760 \mathrm{GPa}$, $K_{3}=334.906 \mathrm{GPa}, \Gamma=1.43$. The parameters for aluminum are $\rho_{0}=2703.8 \mathrm{~kg} / \mathrm{m}^{3}, K_{1}=79.883 \mathrm{GPa}, K_{2}=113.894 \mathrm{GPa}$, $K_{3}=139.757 \mathrm{GPa}, \Gamma=2.09$. The parameters for copper are $\rho_{0}=8944.9 \mathrm{~kg} / \mathrm{m}^{3}, K_{1}=137.185 \mathrm{GPa}, K_{2}=175.134 \mathrm{GPa}$, $K_{3}=564.177 \mathrm{GPa}, \Gamma=1.96$.

The plastic flow stress for tungsten and aluminum was represented by the Johnson-Cook ${ }^{33}$ model given by

$$
\sigma=\left(C_{1}+C_{2} \bar{\varepsilon}_{p}^{N}\right)\left(1+C_{3} \ln \left(\frac{\dot{\bar{\varepsilon}}_{p}}{\dot{\varepsilon}_{0}}\right)\right)\left(1-\left(\frac{\theta}{\theta_{m}}\right)^{M}\right),
$$

where $\dot{\varepsilon}_{0}$ is the reference strain rate and $\theta_{m}$ is the melting temperature. The parameters for tungsten are $C_{1}=1505.8 \mathrm{MPa}$, $C_{2}=176.5 \mathrm{MPa}, N=0.12, C_{3}=0.016, M=1.0, \theta_{\mathrm{m}}$ $=1723 \mathrm{~K}$. The parameters for aluminum are $C_{1}=115.8 \mathrm{MPa}$, $C_{2}=68.95 \mathrm{MPa}, \quad N=0.58, \quad C_{3}=0.016, \quad M=1.13, \quad \theta_{\mathrm{m}}$ $=923 \mathrm{~K}$. The copper flow stress was represented by the MTS model outlined above. The MTS material parameters for copper are given together with those for tantalum in Table I.

\section{Tantalum material parameters}

The plasticity model material parameters for tantalum used in this study were evaluated by Chen and Gray ${ }^{9}$ and
TABLE I. Tantalum and copper material parameter values for Eqs. (10)-(24) for the ductile damage model used in this study.

\begin{tabular}{lcc}
\hline \hline Parameter & $\mathrm{Ta}$ & Copper \\
\hline$\mu_{0}$ & $65.25 \mathrm{GPa}$ & $47.30 \mathrm{GPa}$ \\
$D_{0}$ & $0.38 \mathrm{GPa}$ & $2.4 \mathrm{GPa}$ \\
$\theta_{0}$ & $40 \mathrm{~K}$ & $130 \mathrm{~K}$ \\
$\sigma_{a}$ & $40 \mathrm{MPa}$ & $40 \mathrm{MPa}$ \\
$\hat{\sigma}_{i}$ & $1203,167 \mathrm{MPa}$ & $0 \mathrm{MPa}$ \\
Initial $\hat{\sigma}_{\varepsilon}$ & $0 \mathrm{MPa}$ & $0 \mathrm{MPa}$ \\
$b$ & $2.863 \dot{\mathrm{A}}$ & $2.550 \mathrm{~A}$ \\
$g_{0 i}$ & $0.1236,5.1463$ & $\ldots$ \\
$g_{0 \varepsilon}$ & 1.6 & 1.6 \\
$\dot{\varepsilon}_{0 i}=\dot{\varepsilon}_{0 \varepsilon}=\dot{\varepsilon}_{0_{\varepsilon s}}$ & $10^{7} \mathrm{~s}{ }^{-1}$ & $10^{7} \mathrm{~s}$ \\
$p_{i}$ & $1 / 2$ & $\ldots$ \\
$q_{i}$ & $3 / 2$ & $\ldots$ \\
$p_{\varepsilon}$ & $2 / 3$ & $2 / 3$ \\
$q_{\varepsilon}$ & 1 & 1 \\
$h_{0}$ & $2.0 \mathrm{GPa}$ & $2.37 \mathrm{GPa}$ \\
$\kappa$ & 3 & 1 \\
$\hat{\sigma}_{\varepsilon s_{0}}$ & $350 \mathrm{MPa}$ & $900 \mathrm{MPa}$ \\
$g_{0_{\varepsilon s}}$ & 1.6 & 0.312 \\
$C_{p}$ & $150 \mathrm{~J} / \mathrm{kg} \mathrm{K}$ & $\ldots$ \\
$\rho_{0}$ & $16640 \mathrm{~kg} / \mathrm{m}^{3}$ & $\ldots$ \\
$k$ & $54.4 \mathrm{~W} / \mathrm{m} \mathrm{K}$ & $\ldots$ \\
$\phi_{0}$ & $1 \times 10^{-4}$ & $\ldots$ \\
$\phi_{f}$ & 0.4 & $\ldots$ \\
$q_{1}$ & 1.5 & $\ldots$ \\
$q_{2}$ & 0.3 & $\ldots$ \\
$q_{3}$ & 2.25 & $\ldots$ \\
$\gamma_{0}$ & 0.4 & $\ldots$ \\
$\gamma_{1}$ & 2.9 & $\ldots$ \\
$\gamma_{2}$ & 0.5 & $\ldots$ \\
\hline \hline & & $\ldots$ \\
\hline
\end{tabular}

Maudlin et al. ${ }^{22}$ The material used for this study was taken from the same plate of base stock as in those works. Mason and Maudlin ${ }^{34}$ have characterized the elastic behavior of this plate material. The values of the symmetric elasticity tensor $\underline{\tilde{\mathbf{L}}}$ used in Eq. (4) and the material parameters for the equation of state given in Eqs. (16) and (17) are given in Table II. These equation of state values were arrived at by the use of data from Marsh $^{35}$ and Crockett. ${ }^{36}$

Even upon annealing, tantalum retains residual crystallographic texture and therefore the resulting inelastic behavior is anisotropic. Maudlin et al. ${ }^{22}$ have characterized this anisotropic response through a crystal plasticity derived flow

TABLE II. Tantalum material parameter values for the elasticity tensor $\underline{\tilde{L}}$ given in Eq. (4), and the equation of state given in Eqs. (16) and (17). Note that the values of $K_{2}$ and $K_{3}$ are assigned a value of 0.0 for tensile values of hydrostatic stress.

\begin{tabular}{lccc}
\hline \hline$\tilde{L}_{11}$ & $283.3 \mathrm{GPa}$ & $\tilde{L}_{55}$ & $137.5 \mathrm{GPa}$ \\
$\tilde{L}_{22}$ & $282.6 \mathrm{GPa}$ & $\tilde{L}_{66}$ & $138.2 \mathrm{GPa}$ \\
$\tilde{L}_{33}$ & $283.0 \mathrm{GPa}$ & $K_{1}$ & $189.7 \mathrm{GPa}$ \\
$\tilde{L}_{12}$ & $144.9 \mathrm{GPa}$ & $K_{2}$ & $295.32 \mathrm{GPa}$ \\
$\tilde{L}_{13}$ & $144.5 \mathrm{GPa}$ & $K_{3}$ & $1092.9 \mathrm{GPa}$ \\
$\tilde{L}_{23}$ & $145.2 \mathrm{GPa}$ & $\Gamma$ & 1.60 \\
$\tilde{L}_{44}$ & $139.0 \mathrm{GPa}$ & $\rho_{0}$ & $16640 \mathrm{~kg} / \mathrm{m}^{3}$ \\
\hline \hline
\end{tabular}


surface. This is represented by the quadratic equation given in Eq. (11). The tensor $\underline{\boldsymbol{\alpha}}$ used here is

$$
\underline{\boldsymbol{\alpha}}=\left[\begin{array}{cccccc}
2.23 & -1.23 & -1 & 0 & 0 & 0 \\
-1.23 & 2.23 & -1 & 0 & 0 & 0 \\
-1 & -1 & 2 & 0 & 0 & 0 \\
0 & 0 & 0 & 4.12 & 0 & 0 \\
0 & 0 & 0 & 0 & 4.12 & 0 \\
0 & 0 & 0 & 0 & 0 & 3.35
\end{array}\right] .
$$

Note that since we have assumed axi-symmetry in all simulations, the actual values for $\alpha_{44}=\alpha_{55}=4.12$ have been averaged and the result is used in both positions.

The tantalum parameters for Eqs. (21)-(27), the rate and temperature sensitive flow stress are given in Table I. Note that there are two values listed for the parameters $\hat{\sigma}_{i}$ and $g_{0 i}$. As discovered by empirical evidence presented by Chen and Gray $^{9}$ and Maudlin et al., ${ }^{22}$ the first number is valid up to a value of 0.161 for the normalized activation energy

$$
\left(\frac{k \theta}{\mu b^{3}} \ln \left(\frac{\dot{\varepsilon}_{0 i}}{\dot{\bar{\varepsilon}}_{p}}\right)\right)^{1 / q_{i}}
$$

and the second is valid for values of Eq. (30) in excess of 0.161 . A constant value of thermal conductivity, $k=54.4 \mathrm{~W} /$ $\mathrm{m} \mathrm{K}$ was used in those simulations where thermal transport was included. ${ }^{37}$ The melting temperature for tantalum was taken as $3270 \mathrm{~K}$.

Although nucleation of damage is an important physical process, it is beyond the scope of the present work and we make no attempt to model any damage initiation but rather assume an initial small value of porosity. For pure materials like the tantalum used in this study, this value is expected to be rather small and a value of $\phi_{0}=0.0001$ is assumed here. The values of material parameters for the failure model of Eqs. (14) and (15) were derived from a combination of the work of Mason and Maudlin ${ }^{34}$ and Mason ${ }^{38}$ as well as the tantalum on tantalum experimental results presented here. The numerical results presented with the aluminum/tantalum on tantalum and copper/tungsten on tantalum experiments are predictions without additional parameter adjustment. In particular, all damage model material parameters were evaluated against that experiment using the numerical model with a computational cell size of $150 \mu \mathrm{m}$. The material parameters contained in Eqs. (10)-(15) are listed in Table I.

\section{NUMERICAL}

The explicit finite element code EPIC- $06^{39}$ was used to simulate the deformation response of the plate impact results. The two-dimensional simulations assumed axi-symmetry and preserved the anisotropy of the geometry. Since crosssectional metallography of the recovered samples was an integral part of this study, simulation of the full experimental geometry, with radial momentum trapping rings, was necessary. The exact geometry of the experimental impact and sample plates was replicated numerically. A schematic of the general meshing geometry (without element representation) is given in Fig. 5 and shows the central red sample with alternating red and yellow momentum trapping rings to preserve the integrity of the sample from edge release. The flyer plate composite-composed of either one or two materials-was given the experimentally determined initial velocity and impact between the flyer assembly and initially stationary plate assembly was simulated. Frictionless contact surfaces between each of the independent members of the assembly were assumed and have generally been found to be an accurate assumption for these types of loading conditions.

The question of mesh sensitivity in the context of ductile damage will be addressed to some extent in this work and for each of the simulation configurations; the highest mesh density is focused only in the sample material. The flyers and radial rings are meshed more coarsely. Within the sample regions, the computational cells are composed of square cross-triangular elements of radial and axial dimensions of $25,50,75,100$, and $150 \mu \mathrm{m}$. There are four integration points within each of the square computational cells. The dimensions of the computational cells within the flyer and radial momentum capture regions are not altered and are structured square and on average have an approximate dimension of $100 \mu \mathrm{m}$ in both the in-plane directions. The calculated free surface velocity compared against the experiments is the particle velocity at a free-surface node of the sample very near the center of the model.

Regularization of the equations under the macroscopic softening conditions of damage evolution using internal state variable theories as done here is generally necessary to achieve a numerical solution. ${ }^{40-42}$ Adding viscosity to the equation set is generally a numerical technique to achieve this and is what was done here through the use of the overstress parameter $\tau_{r}$. It should be mentioned that this is in general an unsolved problem for the types of models discussed

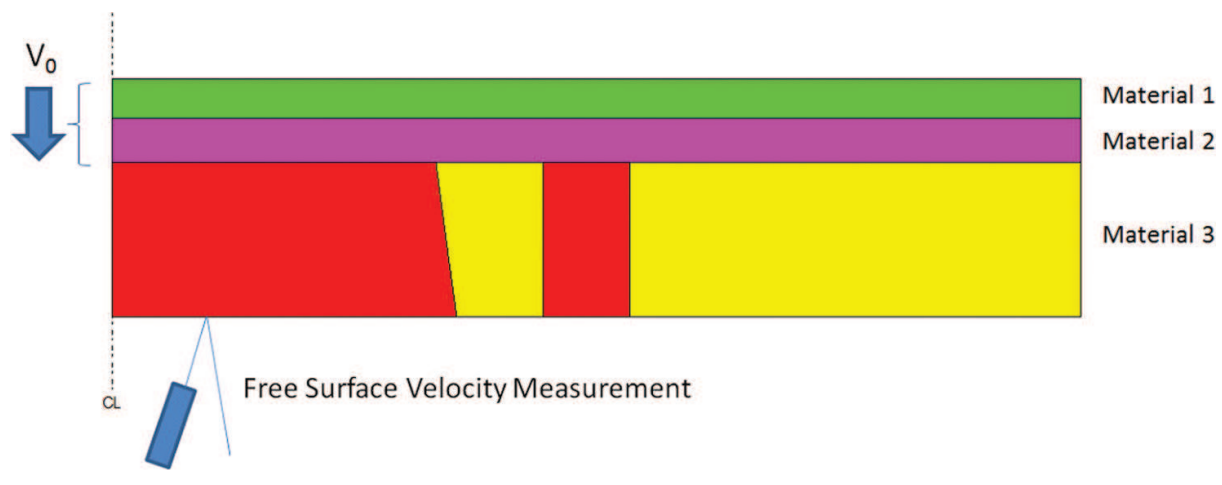

FIG. 5. Schematic drawing of the axisymmetric layered flyer plate configuration. The green and magenta regions comprise the composite flyer plates. The center red region is the recovered sample. The three yellow/red/yellow regions represent the three momentum trapping rings. All calculations performed were $2 \mathrm{D}$ axi-symmetric with the center-line at the far left of the figure. 


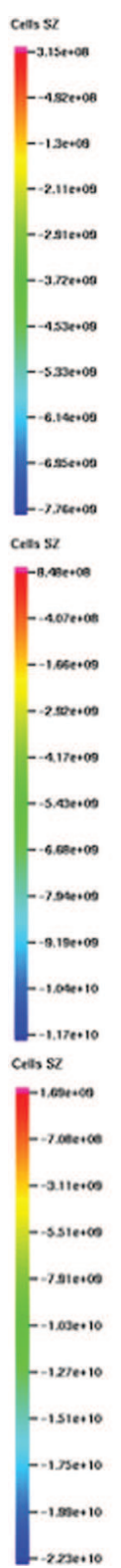

(a)

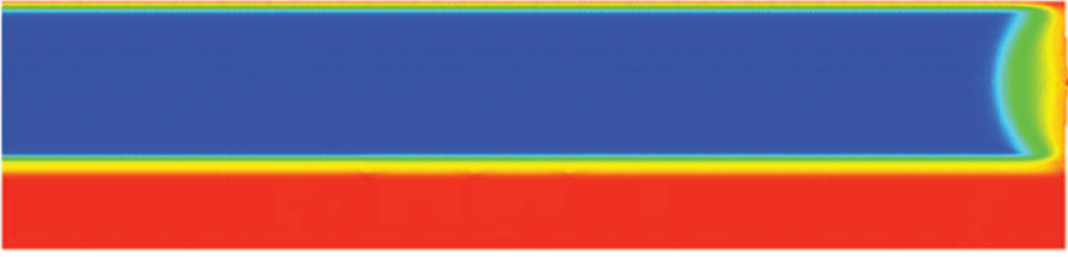

(b)

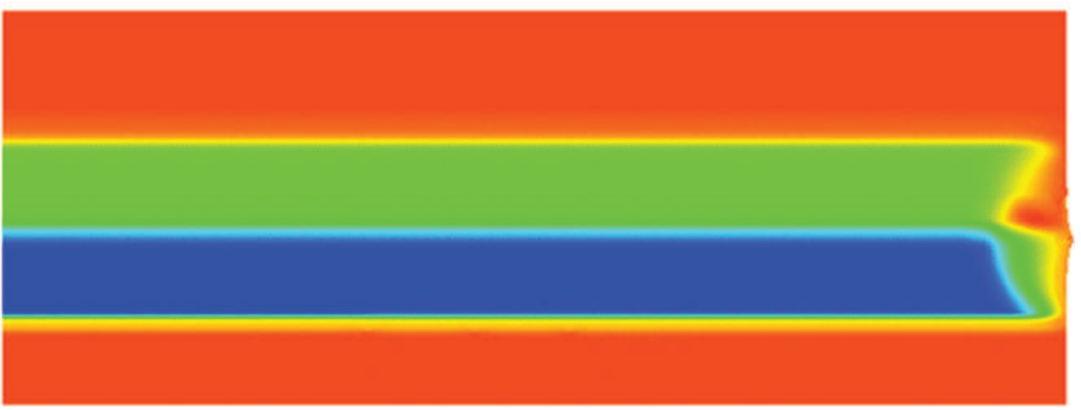

(c)

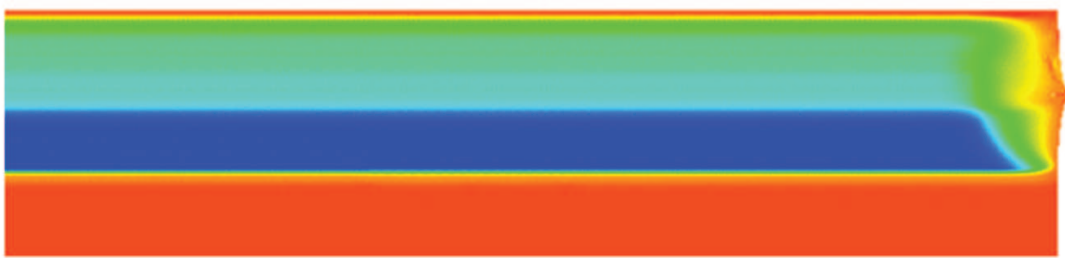

FIG. 6. Contour plots of shock direction component of stress within all three sample assemblies at $0.6 \mu$ s after impact. The shock front is approximately at the center of the sample. The interface between the sample and flyer plate assembly is distinguished by the right hand free edge showing a peak of deformation. All calculations performed were 2D axi-symmetric with the center-line at the far left of the figure. here. Through the addition of viscosity, integral non-local quantities, or spatial gradient quantities to the equations; it still generally remains a challenge to do so in a physically consistent way. Most regularization strategies employed are numerical tools which work to some extent, but the solution is altered in a way, which does not adequately represent the physical system of the material. Through the numerical experience of the three experiments presented here; numerical simulations have suggested that to achieve successful solutions for all model resolutions, the value of $\tau_{r}=27.4$ $\mathrm{N} \mathrm{s} / \mathrm{m}^{2}$ or smaller was necessary. Values of $\tau_{r}$ larger than $27.4 \mathrm{~N} \mathrm{~s} / \mathrm{m}^{2}$ resulted in code instability in the time region of mechanical pull-back of the simulation. In general, the large cell-size problems tolerated larger threshold values of $\tau_{r}$. The value quoted above is that necessary for all mesh density problems to successfully achieve solution to late time (to enable comparison to the cross-sectioned samples). The numerical results reported below are those run with a value of $\tau_{r}=6.8 \mathrm{~N} \mathrm{~s} / \mathrm{m}^{2}\left(l=1 \times 10^{-7} \mathrm{~m}\right)$ to ensure consistent behavior across all material combinations and mesh densities.

Polycrystalline metallic materials are aggregate composites and the way in which materials nucleate and propagate damage of all types is strongly influenced by this heterogeneous nature. Numerical simulations of polycrystalline aggregates have suggested broad stress distributions within this class of materials. ${ }^{28}$ The experimental results and the crosssectional metallographic images of the three samples in this work demonstrate this linkage with the material microstructure. Additional research is required to quantitatively represent the statistical nature of polycrystalline metallic materials in models of damage. This is especially true in the context of macroscale internal state variables as discussed in this work. To this end however, we have performed all the reported calculations with a simple level of statistical variability to ensure that numerical variability does not intervene in determining the morphology of the damage field. For this work, we have used randomized values of $\sigma_{a}$ in Eq. (21) so 
that values range between 0.0 and $80.0 \mathrm{MPa}$ (on average the entire numerical model still has a value of $\sigma_{a}=40 \mathrm{MPa}$ as reported in Table I). We have also randomized the initial value of porosity, $\phi_{0}$, assigned to each computational material point so that values range between 0.0 and $2 \times 10^{-4}$. A similar approach was used by Becker ${ }^{43}$ in the study of ring fragmentation, where it was found that material heterogeneity is particularly important to the resulting fragment field. Each material point in the numerical model for the sample material has a differing value of $\sigma_{a}$ and $\phi_{0}$ within the specified ranges. In the future more sophisticated and physically realistic representations of spatial material variability are envisioned. This will also play a role in developing physically based regularization techniques and strategies that go far beyond what exist today. All numerical results presented here were performed with this spatially randomized $\sigma_{a}, \phi_{0}$ strategy. Overall, this simple step, together with added viscosity $\tau_{r}$, reduced the degree of sensitivity of numerical results due to computational cell size. Although not included in this model, inertial effects of pore growth ${ }^{44}$ are also believed to be relevant for high density materials such as tantalum and also are expected to introduce additional regularization effects through the additional time rate sensitivity of pore growth.

\section{NUMERICAL RESULTS}

Numerical simulation results for the three experimental configurations presented earlier are given in Figs. 6-12. The stress contour plots for all three experimental configurations are given in Fig. 6 showing the stress achieved at the point in time when the shock front is near the center of the sample. Each of the three plots is taken at a time of $0.6 \mu$ s after impact. The results compare favorably with the stress targets reported for each of the three experiments described in Section III A. The tantalum on tantalum free surface velocity results for each of the 5 different mesh density calculations is compared against that of the experiment in Fig. 7. Late time images of the density profile within the numerical simulations at a time of $15 \mu \mathrm{s}$ for both the $150 \mu \mathrm{m}$ and $25 \mu \mathrm{m}$ cell size models are given in Fig. 8. Although the general character of the free-surface velocity profiles is the same between different resolution models, the more highly resolved models retain more energy after first release. This is reflected in the density images of Fig. 8 as well. The $25 \mu \mathrm{m}$ cell size deformed mesh shows clear failure within the center section while the lower density mesh does not. The experimental cross-sectional image suggests that complete failure does not occur within the sample for this experimental configuration. Representation of the size of the damage field is generally reasonable for the numerical simulations, although the damage field width of the lower density model is greater, which may be cause for greater energy dissipation than the high mesh density models. The reader should be reminded that the $150 \mu \mathrm{m}$ model for the tantalum on tantalum configuration was used to evaluate the model material parameters.

The aluminum/tantalum on tantalum numerical results is given in Figs. 9 and 10. The numerical free-surface velocity profiles are nearly identical until a time of approximately

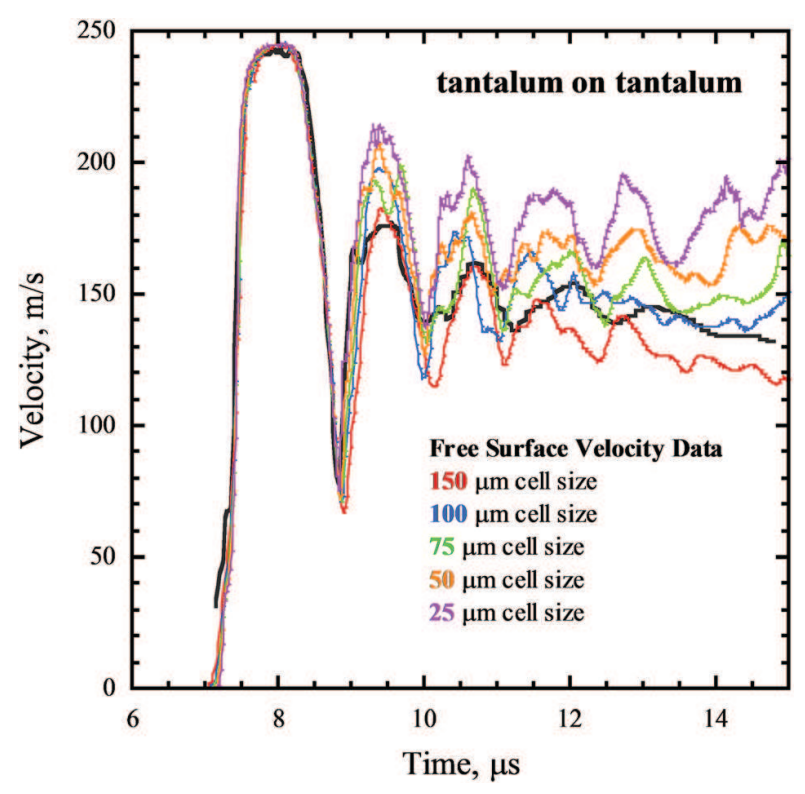

FIG. 7. Free surface velocity traces for the tantalum on tantalum configuration. The black curves are the experimental result, while the colored curves are simulation results with the indicated computational cell size for each indicated. The simulations reported in this figure were for $l=1 \times 10^{-7} \mathrm{~m}$ and randomized $\phi_{0}$ and $\sigma_{a}$.

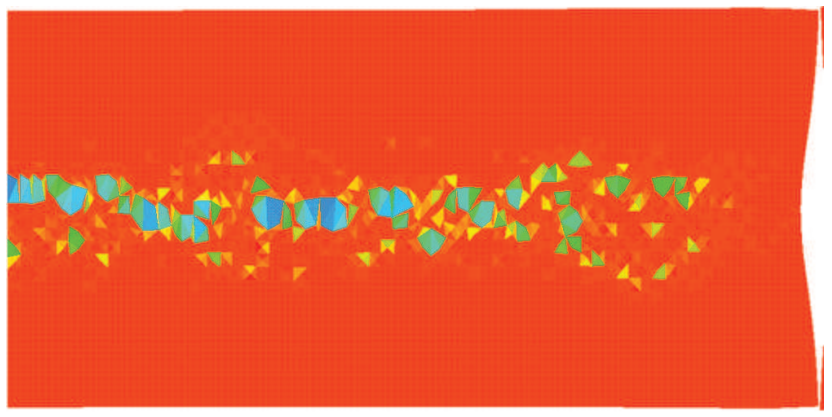

(a)

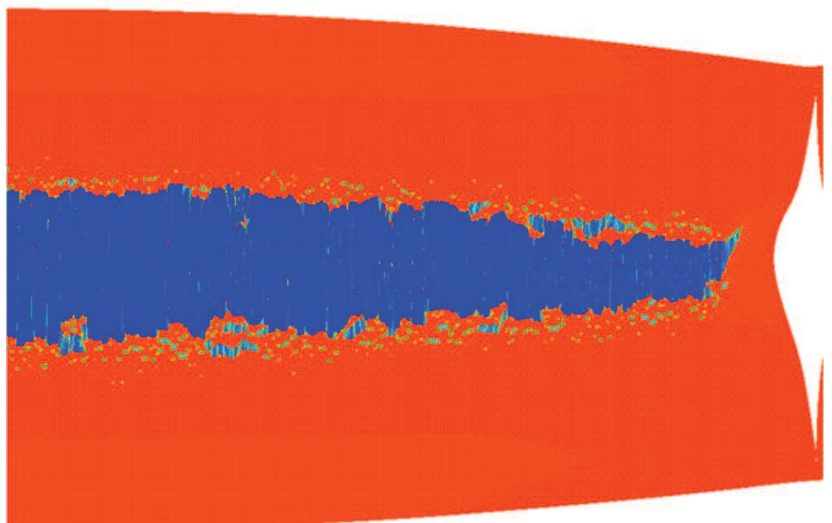

(b)

FIG. 8. Simulation result (density contour) of the sample at late time for the tantalum on tantalum configuration; (a) $150 \mu \mathrm{m}$ cell size; (b) $25 \mu \mathrm{m}$ cell size. The red regions are fully dense while the blue regions are fully failed. The simulations reported in this figure were for $l=1 \times 10^{-7} \mathrm{~m}$ and randomized $\phi_{0}$ and $\sigma_{a}$. These images were taken at a time of $15 \mu \mathrm{s}$. The free surface is at the bottom. 


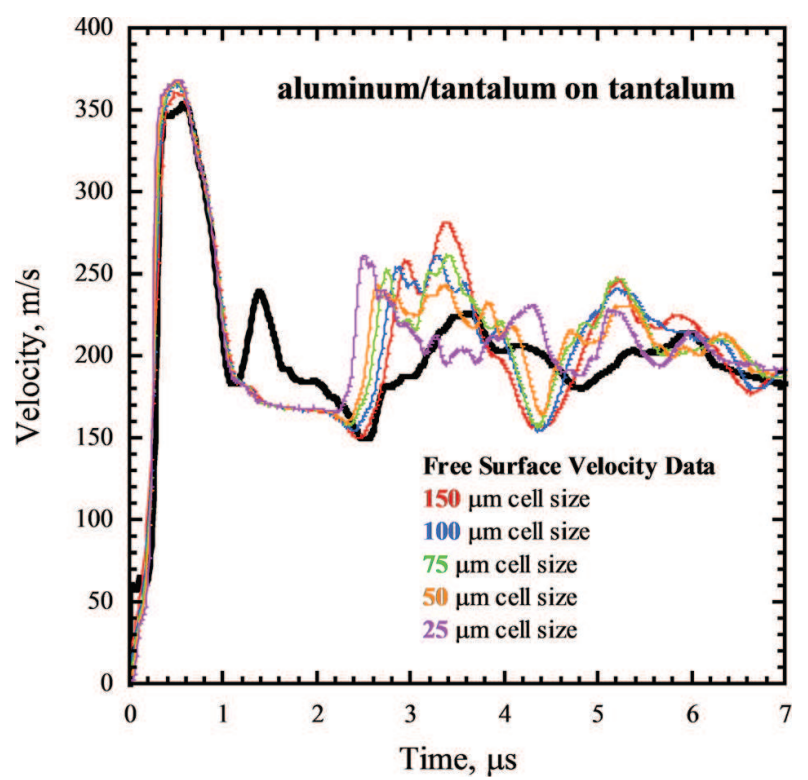

FIG. 9. Free surface velocity traces for the aluminum/tantalum on tantalum configuration. The black curves are the experimental result, while the colored curves are simulation results with the indicated computational cell size for each indicated in the legend. The simulations reported in this figure were for $l=1 \times 10^{-7} \mathrm{~m}$ and randomized $\phi_{0}$ and $\sigma_{a}$.

$2.2 \mu$ s which is likely the time at which the damage growth process begins in earnest for each of the models. The difference in damage progression is clearly seen in the 5 free surface velocity traces as compared with the experimental curve. The first pull-back at $1.05 \mu$ s observed experimentally is

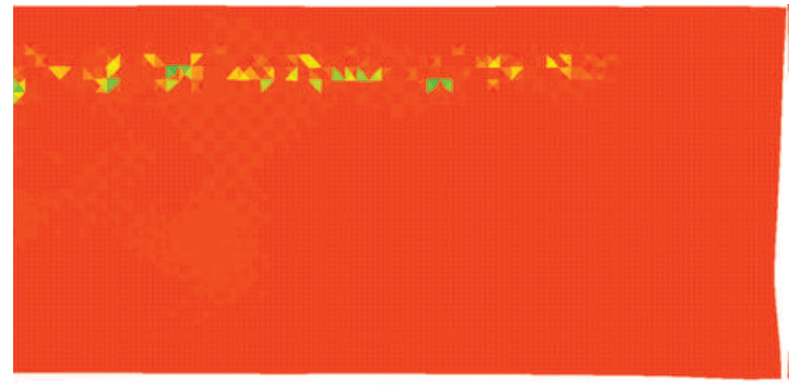

(a)

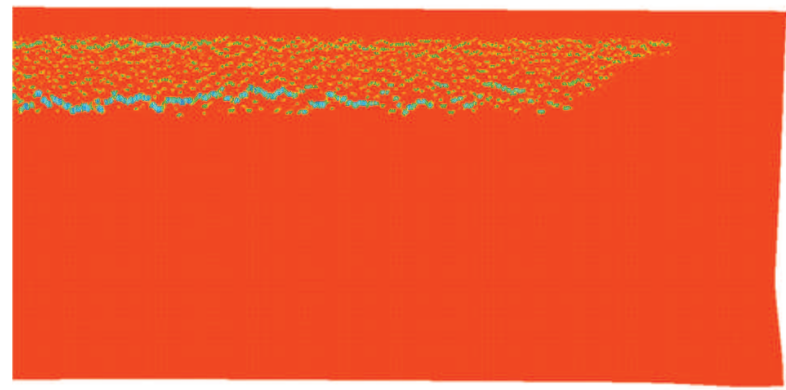

(b)

FIG. 10. Simulation result (density contour) of the sample at late time for the aluminum/tantalum on tantalum configuration; (a) $150 \mu \mathrm{m}$ cell size; (b) $25 \mu \mathrm{m}$ cell size. The red regions are fully dense while the blue regions are fully failed. The simulations reported in this figure were for $l=1 \times 10^{-7} \mathrm{~m}$ and randomized $\phi_{0}$ and $\sigma_{a}$. These images were taken at a time of $15 \mu \mathrm{s}$. The free surface is at the bottom.

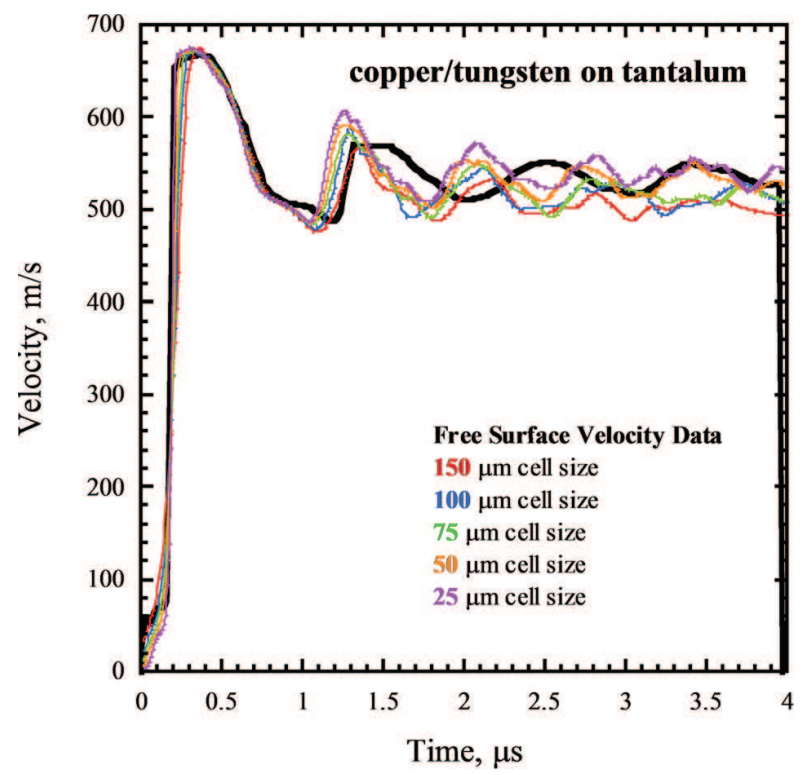

FIG. 11. Free surface velocity traces for the copper/tungsten on tantalum configuration. The black curves are the experimental result while the colored curves are simulation results with the indicated computational cell size for each indicated in the legend. The simulations reported in this figure were for $l=1 \times 10^{-7} \mathrm{~m}$ and randomized $\phi_{0}$ and $\sigma_{a}$.

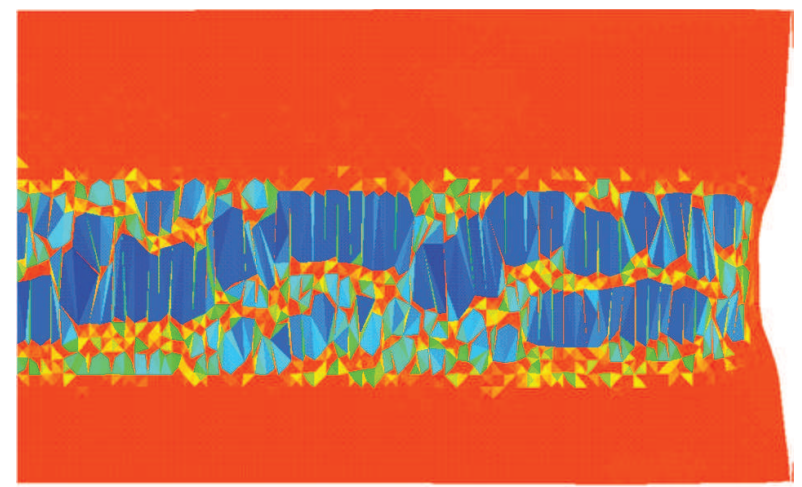

(a)

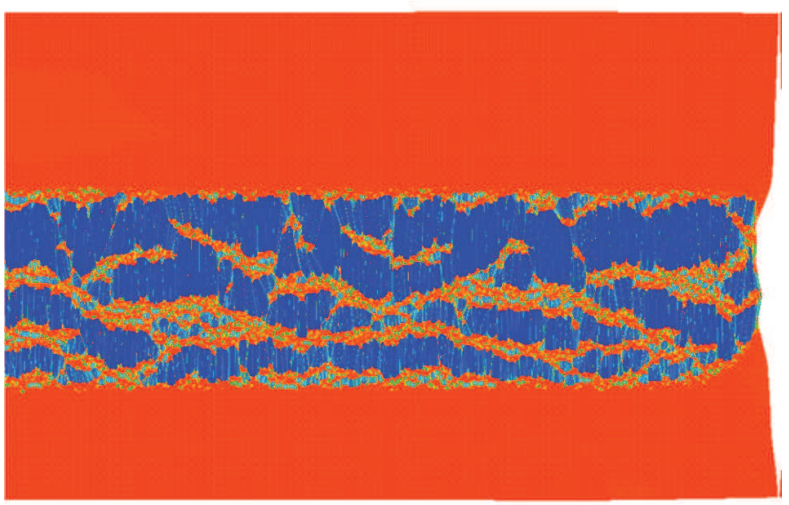

(b)

FIG. 12. Simulation result (density contour) of the sample at late time for the copper/tungsten on tantalum configuration; (a) $150 \mu \mathrm{m}$ cell size; (b) $25 \mu \mathrm{m}$ cell size. The red regions are fully dense while the blue regions are fully failed. The simulations reported in this figure were for $l=1 \times 10^{-7} \mathrm{~m}$ and randomized $\phi_{0}$ and $\sigma_{a}$. These images were taken at a time of $11 \mu \mathrm{s}$. The free surface is at the bottom. 
caused by the damage field developed near the free surface of the sample. This damage region is not predicted by the numerical simulations. Over the course of doing many numerical simulations as part of this study, the second damage region was developed and the proper pull-back response was qualitatively represented. This was a rare event however-as under most conditions this second damage region was not predicted.

The copper/tungsten on tantalum numerical results is given in Figs. 11 and 12. This is clearly an over-driven condition for the tantalum sample as both experimentally and numerically complete failure is readily apparent and there are only small differences between the differing mesh density simulation results. This may be as much due to the fact this the model used in this study does not represent the challenging nucleation process but rather emphasizes the growth and coalescence/failure phase of response. These results emphasize the importance of the nucleation phase of the process-especially under conditions where complete failure is not achieved.

\section{DISCUSSION}

The series of experiments by Gray et al., ${ }^{45}$ and examined in detailed simulations here are an excellent illustration of the strong influence loading profile conditions have upon the damage field produced in polycrystalline metallic materials. The loading profile shape in combination with both the heterogeneous elastic and plastic response of the material combines to result in widely varying dimensions of porosity fields. The most heavily driven of the three conditions - the copper and tungsten flyer at an impact velocity of $616 \mathrm{~m} / \mathrm{s}-$ produced a very narrow region of damage and complete sample separation. In general, the model presented did reasonably well in representing the highest velocity experimental results. Very little mesh sensitivity was demonstrated in the free-surface velocity results and those that were observed occurred at the later stages in the release process. The later time reverberation pattern in the free-surface velocity profile was generally not well represented. This is true in general for all three loading conditions. This later time wave profile is strongly dependent upon the morphological details of the damage field and so the ability to represent these details is still a significant challenge with macro-scale tools as used here. In addition, the loading conditions under shock are not monotonic and most materials demonstrate some degree of the Bauschinger Effect under cyclic loading conditions. This is not represented in the plasticity model used in this study and so could also be an additional source of discrepancy between the wave profiles produced experimentally and numerically.

The tantalum on tantalum loading configuration produced as expected, a symmetric field of damage along the sample center-line. It is notable that this damage field is quite broad, indicating for the relatively slow imposed impact velocity condition of $249 \mathrm{~m} / \mathrm{s}$ a broad and sustained tensile pulse during the release of the material. Examining the numerical results confirms that under the early stages of the release process, indicated by the model when the growth rate of porosity begins to rapidly increase, the tensile pulse experienced by the material is symmetric over the entire sample thickness and is near Gaussian in shape. Experimentally, this sample clearly demonstrates early stage coalescence through localized deformation between pore regions. This is interesting in the context that prior work on the localized shear response of materials indicated the strong resistance of tantalum to form adiabatic shear bands under forced shear conditions. ${ }^{26}$ The forced shear experiments were performed on materials from their initially annealed condition-very different from the material state under which localizations are observed here. It has generally been observed that first shock passage through the material evolves the structure of the material such that measures of plastic flow stress are substantially different from the starting material-even though the amount of plastic deformation is believed to be relatively small. ${ }^{8,21,46-49}$ Although we do not understand the details of specific structural changes occurring during first shock, it is likely that the dislocation structure and even mechanical twinning take place during that period of first rapid loading. This shock pre-conditioning has been demonstrated to substantially increase the propensity of the material to form localized regions of shear. In addition, the material surrounding the pores has experienced a great deal of plastic deformation and produced a substantial amount of stored internal energy by the time the coalescence process begins through localization. It is interesting to note that, although the model performed reasonably well in representing the tantalum on tantalum response, this condition also produced the greatest degree of mesh sensitivity as demonstrated by the free-surface velocity results. The simulation results presented in Fig. 7 give an indication to what is perhaps the cause of this. The higher resolution demonstrates much larger separation-indicating that the lower resolution calculation remains intact for longer times and is able to absorb a greater amount of energy due to plastic deformation compared with the higher resolution case. The smaller cells are believed to facilitate earlier localized deformation and therefore satisfy the failure criterion earlier in time than when deformation is averaged over larger computational cells. This perhaps suggests that the complexity of late stage pore growth together with coalescence is not adequately represented by the simple coalescence/failure criterion used here.

The final of the three experimental configurations is that of the aluminum and tantalum flyer imposed at an impact velocity of $350 \mathrm{~m} / \mathrm{s}$. This condition produced two distinct damage regions, each of which is near both the impact and free surfaces. The upper damage region is clearly more developed as the detailed metallography in Figs. 2-4 clearly illustrate. The metallographic images in Figs. 2(b) and 3(b) indicate reasonably similar spatial density in pores as best can be discerned from two images, although the upper region has clearly experienced more pore growth. The tomographic images in Fig. 4(a) illustrate this point most clearly. The lower damage region is still in the nucleation dominated region of response, whereas the upper region is clearly established within the pore growth phase of deformation history. The calculations of this condition support this observation in that there is an upper region of a well-established field of damage, 
whereas there is no indication of the lower field being established. Although our introduction here of some degree of spatial variability to represent material initial conditions did over-ride the influence of numerical variability to establishing of the damage field, a more accurate model for linking the structure of the material to pore nucleation is clearly needed. This still remains a significant challenge in general. These calculations did once again display some degree of mesh sensitivity at the later time, but once again the results of Fig. 6 indicate that it is likely due to deficiency of representing the later stages of damage as a much greater degree of surface separation took place in the higher resolution calculation.

\section{CONCLUSION}

We have presented here a study of the shock loading response of tantalum to three different loading conditions using combined material flyer plates to alter the structure of the shock wave within the sample material. The results clearly demonstrate the significance of loading conditions to the way in which polycrystalline metallic material respond to varying shock loading conditions. These three experimental conditions have collectively spanned the regions of nucleation, growth, coalescence, and failure for the porosity based damage response of polycrystalline metallic materials. Each of the samples and regions within samples, spanned different combinations of early to late stage damage to produce an excellent challenge for numerical representation. A model for the finite elastic, plastic, and damage response of polycrystalline metallic materials was used to numerically simulate each of the three experimental conditions. Overall, the model performed well for regions of response which were dominated by porosity growth, but performed less well when nucleation or coalescence plays prominent roles. These were indicated weaknesses in the material model and also point to significant challenges as the physical processes dominating both of these responses are strongly influenced by the structure of the material. It remains a challenge, how best to represent material microstructure in models for the macro-scale representation of damage for large-scale engineering applications.

\section{ACKNOWLEDGMENTS}

Los Alamos National Laboratory is operated by LANS, LLC, for the NNSA of the U.S. Department of Energy under Contract No. DE-AC52-06NA25396. This research was supported under the auspices of the U.S. Department of Energy and the Joint DoD/DOE Munitions Program (Dr. T. Mason, program manager) as well as DOE Advanced Simulation and Computing Program (Dr. M. Schraad, program manager).

${ }^{1} \mathrm{~J}$. S. Rinehart and J. Pearson, Behavior of Metals under Impulsive Loads (American Society for Metals, Cleveland, OH, 1954).

${ }^{2}$ J. N. Johnson, "Dynamic fracture and spallation in ductile solids," J. Appl. Phys. 52, 2812 (1981).

${ }^{3}$ M. A. Meyers and C. T. Aimone, "Dynamic fracture (spalling) of metals," Prog. Mater. Sci. 28, 1-96 (1983).

${ }^{4}$ G. T. Gray III, N. K. Bourne, and B. L. Henrie, "On the influence of loading profile upon the tensile failure of stainless steel," J. Appl. Phys. 101, 093507 (2007).
${ }^{5}$ J. S. Rinehart, "Scabbing of metals under explosive attack: Multiple scabbing," J. Appl. Phys. 23, 1229 (1952).

${ }^{6}$ B. M. Butcher, L. M. Barker, D. E. Munson, and C. D. Lundergan, "Influence of stress history on time-dependent spall in metals," AIAA J. 2, 977-990 (1964).

${ }^{7}$ J. P. Escobedo, E. N. Brown, C. P. Trujillo, E. K. Cerreta, and G. T. Gray III, "The effect of shock-wave profile on dynamic brittle failure," J. Appl. Phys. 113, 103506 (2013).

${ }^{8}$ G. T. Gray III, "High-strain-rate deformation: mechanical behavior and deformation substructures induced," Annu. Rev. Mater. Res. 42, 285-303 (2012).

${ }^{9}$ S. R. Chen and G. T. Gray III, "Constitutive behavior of tantalum and tantalum-tungsten alloys," Metall. Mater. Trans. A 27A, 2994-3006 (1996).

${ }^{10}$ J. N. Johnson, G. T. Gray III, and N. K. Bourne, "Effect of pulse duration and strain rate on incipient spall fracture in copper," J. Appl. Phys. 86, 4892-4901 (1999).

${ }^{11}$ F. L. Addessio and J. N. Johnson, "Rate-dependent ductile failure model," J. Appl. Phys. 74, 1640-1648 (1993).

${ }^{12} \mathrm{~A}$. L. Gurson, "Continuum theory of ductile rupture by void nucleation and growth: Part 1-yield criteria and flow rules for porous ductile media," J. Eng. Mater. Technol. 99, 2-15 (1977).

${ }^{13}$ P. J. Maudlin, E. N. Harstad, T. A. Mason, Q. H. Zuo, and F. L. Addessio, "TEPLA-a: Coupled anisotropic elastoplasticity and damage," The Joint DoD/DOE Munitions Technology Program progress report, LA-UR14015-PR (2003).

${ }^{14} \mathrm{Q}$. H. Zuo and J. R. Rice, "An implicit algorithm for a rate-dependent ductile fracture model," J. Appl. Phys. 104, 083526 (2008).

${ }^{15}$ G. T. Gray III, V. Livescu, E. K. Cerreta, T. A. Mason, P. J. Maudlin, and J. A. Bingert, "Influence of shockwave obliquity on deformation twin formation in Ta," in DYMAT 2009: 9th International Conference on the Mechanical and Physical Behaviour of Materials Under Dynamic Loading (EDP Sciences, Brussels, Belgium, 2009), p. 963.

${ }^{16}$ B. J. Jensen, D. B. Holtkamp, P. A. Rigg, and D. H. Dolan, "Accuracy limits and window corrections for photon Doppler velocimetry," J. Appl. Phys. 101, 013523 (2007).

${ }^{17} \mathrm{G}$. T. Gray III, "Shock wave testing of ductile materials," in $A S M$ Handbook (Materials Park, Ohio, 2000).

${ }^{18}$ B. L. Adams, S. I. Wright, and K. Kunze, "Orientation imaging-the emergence of a new microscopy,” Metall. Trans. A 24A, 819-831 (1993).

${ }^{19}$ S. A. Novikov, I. I. Divnov, and A. G. Ivanov, "Failure of steel, aluminum and copper under explosive shock loading," Phys. Met. Metall. 21, 122-128 (1966).

${ }^{20} \mathrm{G}$. I. Kanel, "Distortion of the wave profiles in an elastoplastic body upon spalling," J. Appl. Mech. Tech. Phys. 42, 358 (2001).

${ }^{21}$ G. T. Gray III and K. S. Vecchio, "Influence of peak pressure and temperature on the structure/property response of shock-loaded Ta and Ta-10W," Metall. Mater. Trans. A 26A, 2555 (1995).

${ }^{22}$ P. J. Maudlin, J. F. Bingert, J. W. House, and S. R. Chen, "On the modeling of the Taylor cylinder impact test for orthotropic textured materials: Experiments and simulations," Int. J. Plast. 15, 139-166 (1999).

${ }^{23} \mathrm{Q}$. H. Zou, "Modified formulation of a rate-dependent damage model for ductile materials," J. Appl. Phys. 107, 053513 (2010).

${ }^{24} \mathrm{~J}$. W. Hancock and A. C. Mackenzie, "On the mechanisms of ductile failure in high-strength steels subjected to multi-axial stress-states," J. Mech. Phys. Solids 24, 147-169 (1976).

${ }^{25}$ S. Nemat-Nasser and J. B. Isaacs, "Direct measurement of isothermal flow stress of metals at elevated temperatures and high strain rates with application to Ta and Ta-W alloys," Acta. Mater. 45, 907-919 (1997).

${ }^{26} \mathrm{M}$. Kothari and L. Anand, "Elasto-viscoplastic constitutive equations for polycrystalline metals: application to tantalum,” J. Mech. Phys. Solids 46, 51-83 (1998).

${ }^{27}$ C. A. Bronkhorst, E. K. Cerreta, Q. Xue, P. J. Maudlin, T. A. Mason, and G. T. Gray III, "An experimental and numerical study of the localization behavior of tantalum and stainless steel," Int. J. Plast. 22, 1304-1335 (2006).

${ }^{28}$ C. A. Bronkhorst, B. L. Hansen, E. K. Cerreta, and J. F. Bingert, "Modeling the microstructural evolution of metallic polycrystal materials under localization conditions," J. Mech. Phys. Solids 55, 2351-2383 (2007).

${ }^{29}$ U. F. Kocks, A. S. Argon, and M. F. Ashby, "Thermodynamics and kinetics of slip," in Progress in Materials Science (Pergamon, Oxford, 1975).

${ }^{30} \mathrm{P}$. S. Follansbee and U. F. Kocks, "A constitutive description of the deformation of copper based on the use of the mechanical threshold stress as an internal state variable," Acta Metall. 36, 81-93 (1988). 
${ }^{31}$ Y. P. Varshni, "Temperature dependence of the elastic constants," Phys. Rev. B 2, 3952-3958 (1970).

${ }^{32}$ U. F. Kocks, "Laws for work-hardening and low-temperature creep," J. Eng. Mater. Technol. 98, 76-85 (1976).

${ }^{33} \mathrm{G}$. R. Johnson and W. H. Cook, "A constitutive model and data for metals subjected to large strains, high strain rates, and high temperatures," in Seventh International Symposium on Ballistics (The Hague, The Netherlands, April 1983).

${ }^{34}$ T. A. Mason and P. J. Maudlin, "Effects of higher-order anisotropy elasticity using textured polycrystals in three-dimensional wave propagation problems," Mech. Mater. 31, 861-882 (1999).

${ }^{35}$ LASL Shock Hugoniot Data, edited by S. P. Marsh (Univ. Calif. Press, Berkeley, CA, 1980), p. 136.

${ }^{36}$ S. Crockett, private communication (2013).

${ }^{37}$ Metals Handbook, Desk edition, edited by H. E. Boyer and T. L. Gall (ASM, Metals Park, OH, 1985).

${ }^{38}$ T. A. Mason, private communication (2004).

${ }^{39}$ G. R. Johnson, S. R. Beissel, C. A. Gerlach, R. A. Stryk, T. J. Holmquist, A. A. Johnson, S. E. Ray, and J. J. Arata, User Instructions for the 2006 Version of the EPIC Code (Network Computing Services Inc., Minneapolis, MN, 2006).

${ }^{40}$ T. Belytschko, W. K. Liu, B. Moran, and K. I. Elkhodary, Nonlinear Finite Elements for Continua and Structures (John Wiley \& Sons, West Sussex, UK, 2014).

${ }^{41}$ F. R. Ahad, K. Enakoutsa, K. N. Solanski, and D. J. Bammann, "Nonlocal modeling in high-velocity impact failure of 6061-T6 aluminum," Int. J. Plast. 55, 108-132 (2014).
${ }^{42}$ L. Anand, O. Aslan, and S. A. Chester, "A large-deformation gradient theory for elastic-plastic materials: Strain softening and regularization of shear bands," Int. J. Plast. 30-31, 116-143 (2012).

${ }^{43}$ R. Becker, "Ring fragmentation predictions using the Gurson model with material stability conditions as failure criteria," Int. J. Solids Struct. 39, 3555-3580 (2002).

${ }^{44}$ C. Czarnota, N. Jacques, S. Mercier, and A. Molinari, "Modeling of dynamic ductile fracture and application to the simulation of plate impact tests on tantalum," J. Mech. Phys. Solids 56, 1624-1650 (2008).

${ }^{45}$ G. T. Gray III, N. K. Bourne, V. Livescu, C. P. Trujillo, S. MacDonald, and P. Withers, "The influence of shock-loading path on the spallation response of Ta," in Proceedings of APS Topical Group of Shock Compression of Condensed Matter, Seattle, 7-12 July 2013.

${ }^{46}$ S. A. Maloy, G. T. Gray III, C. M. Cady, R. W. Rutherford, and R. S. Hixson, "The influence of explosive-driven "Taylor-wave" shock prestraining on the structurelproperty behavior of 304 stainless steel," Metall. Mater. Trans. A 35A, 2617 (2004).

${ }^{47}$ B. H. Sencer, S. A. Maloy, and G. T. Gray III, "The influence of explosive-driven shock prestraining at $35 \mathrm{GPa}$ and of high deformation on the structure/property behavior of 316L austenitic stainless steel," Metall. Mater. Trans. A 36A, 1825 (2005).

${ }^{48}$ B. H. Sencer, S. A. Maloy, and G. T. Gray III, "The influence of shockpulse shape on the structure/property behavior of copper and $316 \mathrm{~L}$ austenitic stainless steel," Acta Mater. 53, 3293 (2005).

${ }^{49}$ Q. Xue, G. T. Gray III, B. L. Henrie, S. A. Maloy, and S. R. Chen, "Influence of shock prestraining on the formation of shear localization in 304 stainless steel,” Metall. Mater. Trans. A 36A, 1471 (2005). 